\title{
AMH as a Prognostic Factor for Blastocyst Development
}

\author{
Emily De Conto ${ }^{1,2}$, Vanessa Krebs Genro ${ }^{2,3}$, Daniela Scherer da Silva ${ }^{1,2}$, Rita de Cassia Borges Chapon ${ }^{1,2}$, João \\ Sabino Lahorgue Cunha Filho, ${ }^{1,2}$
}

${ }^{1}$ Universidade Federal do Rio Grande do Sul, Porto Alegre/RS, Brazil

${ }^{2}$ INSEMINE Center of Human Reproduction, Porto Alegre/RS, Brazil

${ }^{3}$ Hospital de Clínicas de Porto Alegre, Porto Alegre/RS, Brazil

\begin{abstract}
Objective: To investigate the relationship between $\mathrm{AMH}$ blood levels and the likelihood of blastocyst formation.

Methods: Two hundred ninety-two patients, 22-44 years of age, undergoing routine explorations during spontaneous cycles that preceded assisted reproductive technologies at our Center, were studied. As the present study did not require previous submission to our Institutional Review Board. Serum AMH and FSH levels were measured and laboratory data was obtained after ovulation induction with an antagonist protocol. Participants were sorted into two different groups paired by age. The first group (No Blasto; $n=219$ ) involved women having no blastocyst formation; the second group (Yes Blasto group; $n=73$ ) was made up of those women who were considered eligible to undergo 5 days of embryo culture. Furthermore, we analyzed blastulation rate. Patients were divided according to the rate of blastocyst formation $<0.43(n=36)$ and $\geq 0.43(n=37)$. The Statistical analysis was performed using SPSS version 20.0. We ran Student's t-test for independent samples and Pearson's correlation. A $P<0.05$ was considered significant.
\end{abstract}

Results: AMH levels were statistically different $(P=0.002)$ between the YES and NO blasto groups. Number of oocytes, MII oocytes and embryos were higher in Yes Blasto group. FSH levels were similar between the groups $(P=0.149)$. Pearson correlation coefficient shows that the rate of blastocyst formation is inversely correlated to $\mathrm{AMH}$ levels.

Conclusions: We conclude that patients that were considered eligible to undergo blastocyst formation have higher levels of serum $\mathrm{AMH}$, however too high concentration of this hormone can be harmful to blastocyst development.

Keywords: $\mathrm{AMH}$, Blastocyst, Prognostic factor, IVF

\section{INTRODUCTION}

The search for prognostic factors in reproductive cycles has been an issue of constant debate in the literature. Anti-Mullerian Hormone (AMH) is one of the topics of research in this field since its levels are better able to predict ovarian response to gonadotropins in IVF cycles than FHS levels, or even age itself. (Satwik et al., 2012). AMH levels reflect the patient's ovarian reserve and it is a good marker of gonadotropin dose requirements in GNRH agonist cycles (Anckaert et al., 2012).

Some authors have correlated AMH levels with the number of oocytes and MII oocytes retrieved in IVF oocyte retrieval (Lin et al., 2013); others, however, have demonstrated that it is neither related to oocyte quality (Guerif et al., 2009), nor to number of embryos (Smeenk et al., 2007). Controversial results are found in the literature when it comes to AMH levels and pregnancy likelihood. It has been shown that its levels are not well correlated to embryo quality or pregnancy likelihood (Smeenk et al., 2007); however, a more recent study states that AMH is a good prognostic factor for IVF pregnancy outcome (Kamel et al., 2014). Studies have also shown that blood AMH levels can help predict the likelihood of blastocyst formation (Lin et al., 2013; Sills et al., 2011). We believe that all these associations should be further investigated.

Blastocyst formation is a fundamental step for IVF result optimization, since this is the physiological stage of embryo development in uterus (de los Santos et al., 2003) and would be logical to transfer only blastocysts whenever possible. Moreover, the evaluation and the choice of the embryo to be transferred is easier and more accurate with longer in vitro time for embryo development (Langley et al., 2001). Thus, the best embryo in the most appropriate stage of development shall be deposited in the best place for nesting, and these factors indeed influence the success of assisted reproduction treatment.

Our study was designed to investigate the relationship between AMH blood levels and the likelihood of blastocyst formation.

\section{MATERIAL AND METHODS \\ Subjects}

Two hundred ninety-two patients, 22-44 years of age, undergoing routine explorations during spontaneous cycles that preceded assisted reproductive approaches at our Center, were studied. All patients met the following inclusion criteria: (i) regular, ovulatory menstrual cycles every 25-35 days; (ii) both ovaries present; (iii) no clinical signs of hyperandrogenism; (iv) no current or past diseases affecting ovaries or gonadotropin, or sex steroid secretion, clearance or excretion; (v) no current hormone therapy. Patients with severe male factor infertility were excluded. As the present study was merely observational and included only data from routine measurements, it did not require submission to our Institutional Review Board.

\section{Hormonal Measurements and Laboratory Results}

On cycle day 3, each woman underwent a blood sampling by venipuncture for serum AMH and FSH levels. Laboratory data was obtained after ovulation induction with antagonist protocol.

Serum AMH levels were determined using an ultrasensitive enzyme-linked immunosorbent assay (ELISA). Intraand inter-assay coefficients of variation were less than $6 \%$ and less than $10 \%$, respectively, the lowest detection limit was at $0.13 \mathrm{ng} / \mathrm{mL}$, and linearity was up to $21 \mathrm{ng} / \mathrm{mL}$. Serum levels of FSH were determined using an automated multi-analysis system with chemiluminescence detection.

Embryo evaluation was performed according to Fisch et al. (2001) criteria. The first post-fertilization assessment (16-18 hours after insemination) takes into account the alignment of nucleoli along the pronuclear axis. The second evaluation (25-27 hours after insemination) notes the regularity and symmetry of cleavage and presence or absence of fragmentation. The third observation (64-67 hours after insemination) checks the number and regularity of blastomere size and presence or absence of fragmentation. 
In the fourth and last review, the blastocyst formation is observed according to criteria determined by Gardner et al. (2004): size of blastocele, cell number and cohesion of the inner cell mass and trophectoderm. Patients who have had at least three embryos with scores equal to or greater than eighty points according to Fisch et al. (2001) on day three were considered eligible to undergo 5 days of embryo culture.

\section{Definition of Groups}

To study the different baseline features between patients who were considered eligible to blastocyst development, the participants were sorted into two different groups, paired by age.

The first group (No Blasto; $n=219$ ) was made up of women having fewer than two embryos or less than $3 \mathrm{em}-$ bryos with more than 80 points according to Fisch et al. (2001); the second group (Yes Blasto group; $n=73$ ) was made up of those presenting at least one blastocyst after 5 days of embryo culture. Furthermore, to evaluate factors associated to blastocyst development, the patients were divided into rate of blastocyst formation $<0.43(n=36)$ and $\geq 0.43(n=37)$.

The blastocyst formation rate was calculated by dividing the number generated by the number of blastocysts and embryos ranged from 0 to 1 , the median (0.43) was used to differentiate cycles at higher and lower then blastocyst formation.

\section{Statistical Analysis}

The Statistical analysis was performed using the Statistical Package for Social Sciences (SPSS Inc., Chicago, Illinois, USA) version 20.0. We used the Student's t-test for independent samples and the Pearson's correlation. A $P<$ 0.05 was considered significant.

\section{RESULTS}

Baseline results are depicted on Table 1 . Since patients were paired by age, their mean age was similar between the groups. Gonadotropin initial doses for ovulation induction were significantly different. Number of oocytes, MII oocytes and embryos were significantly higher in the Yes Blasto group. FSH levels were similar between the groups $(P=0.149)$; however, AMH levels were statistically different $(P=0.002)$.

The Pearson's correlation coefficient between AMH levels and blastocyst formation was -.234 $(P=0.046)$, which shows a linear relationship between these variables and establish statistical dependence between them.

Table 2 shows that the rate of blastocyst formation is inversely correlated to AMH levels. This data shows that in a selected population of women with good ovarian response the fact of presenting high AMH levels can impair embryo development.

\section{DISCUSSION}

Our results showed that the group considered eligible to blastocyst formation had significantly higher serum $\mathrm{AMH}$ levels and higher initial gonadotropin dose during ovulation induction and similar FSH levels. In addition, this group also presented with more oocytes retrieved, more MII oocytes and more embryos generated. These results were not statistically significant; however they are in agreement with data found in the literature. However, the most important and significant finding of this study is the fact that serum AMH concentration can be used as a prognostic factor for blastocyst formation.

According to in vitro culture assessments, 73 cycles were considered competent for blastocyst formation and its culture has been extended to Day 5 post-puncture. We found that the group with the higher number of blastocysts had lower concentrations of AMH, fewer oocytes/MII oocytes retrieved and less generated embryos. This may be a result of an erratic recruitment of follicles in hormonal induction: the higher the number of follicles, the greater the likelihood of incompetent oocytes having abnormal nuclear or incomplete cytoplasmic maturation; abnormal distribution of cytoplasmic organelles, thus presenting failures in fertilization or blocking in embryonic development. It has been shown by others that AMH and the chromosomal competence of embryos are not correlated (Lie et al., 2008).

This erratic follicular recruitment indirectly seen in our findings in women with high levels of AMH can be explained by an increase in aneuploidy rates in embryos. Women with higher AMH serum levels presented an inferior embryonic development (blastulation). Since AMH has an intimate relationship with number of oocytes retrieved (Broekmans et al., 2006), a goal has to be set that provides the lab with a sufficient number of good-quality eggs but does not put the patient at risk for hyperstimulation. Several studies have evaluated the "optimal" egg number. Steward et al. (2014) used the Society for Assisted Reproductive Technology registry from 2008 to 2010 to study live birth rates and ovarian hyperstimulation syndrome (OHSS) rates based on 256,381 cycles. There was a continuous increase in live birth rates across the egg categories up until 11-15 oocytes (0-5 oocytes: $17 \% ; 11-15$ oocytes: $39.3 \%$ ) (Steward et al., 2014). There was, however, no further significant increase in live birth rates in the higher oocyte yield groups. Receiver operating characteristic analysis determined 15 oocytes as a cut-off above which OHSS risk significantly increases (Steward et al., 2014).

Higher egg yield is associated with benefits. On the other hand, the simultaneous development of several follicles will result in supraphysiologic steroid hormone levels that could have an adverse effect on implantation, placentation, and even perinatal outcome (Evans et al., 2014). The risk of OHSS, which is a potentially life-threatening complication of ovarian stimulation, also increases with

Table 1. Description of Yes and No blasto groups

\begin{tabular}{|l|c|c|c|}
\hline & No Blasto $(\boldsymbol{n = 2 1 9 )}$ & Yes Blasto (n=73) & P-value* \\
\hline Age (years) & $34.4 \pm 2.3$ & $34.3 \pm 4.0$ & 0.891 \\
\hline FSH (IU/L) & $7.89 \pm 2.9$ & $7.16 \pm 2.3$ & 0.149 \\
\hline AMH (pmol/L) & $3.45 \pm 3.1$ & $4.86 \pm 3.9$ & 0.002 \\
\hline Gonadotropin initial dose (IU/L) & $264 \pm 54$ & $248 \pm 63$ & 0.039 \\
\hline Number of Oocytes retrieved & $5.33 \pm 3.7$ & $9.01 \pm 4.9$ & $<0.001$ \\
\hline Number of MII oocytes & $4.41 \pm 3.1$ & $8.15 \pm 4.5$ & $<0.001$ \\
\hline Number of embryos & $2.47 \pm 1.8$ & $6.63 \pm 3.8$ & $<0.001$ \\
\hline
\end{tabular}

Mean \pm standard deviation

FSH: Follicle Stimulating Hormone, AMH: Anti-Mullerian Hormone *Student's t Test 
Table 2. Groups with different rates of blastocyst formation

\begin{tabular}{|l|c|c|c|}
\hline Blastocyst rate development & $\mathbf{< . 4 3}(\mathbf{n = 3 6 )}$ & $\mathbf{2 0 . 4 3}(\mathbf{n = 3 7})$ & $\boldsymbol{P}$-value* \\
\hline Age (years) & $33.8 \pm 4.6$ & $34.8 \pm 3.3$ & 0.287 \\
\hline FSH (IU/L) & $6.81 \pm 1.3$ & $7.53 \pm 3.0$ & 0.323 \\
\hline AMH (pmol/L) & $6.05 \pm 4.7$ & $3.71 \pm 2.4$ & 0.011 \\
\hline Gonadotropin initial dose (IU/L) & $244 \pm 68$ & $251 \pm 60$ & 0.637 \\
\hline Number of Oocytes retrieved & $10.03 \pm 5.5$ & $8.03 \pm 4.0$ & 0.081 \\
\hline Number of MII oocytes & $8.83 \pm 5.2$ & $7.49 \pm 3.6$ & 0.199 \\
\hline Number of embryos generated & $7.22 \pm 4.4$ & $6.05 \pm 3.2$ & 0.197 \\
\hline Number of blastocysts & $1.89 \pm 1.7$ & $3.78 \pm 1.8$ & $<0.001$ \\
\hline Rate blastulation & $0.23 \pm 0.12$ & $0.67 \pm 0.19$ & $<0.001$ \\
\hline
\end{tabular}

Mean \pm standard deviation

FSH: Follicle Stimulating Hormone, AMH: Anti-Mullerian Hormone

*Student's t Test

the number of oocytes collected (Steward et al., 2014). Most studies put the optimal number of retrieved oocytes between 10 and 15 (Briggs et al., 2015; van der Gaast et al., 2006; Sunkara et al., 2011). Even in the currently discussed study, the increase was less pronounced over 15 oocytes.

Since it is an observation study, it implies no randomization. On the other hand, all our patients underwent the same ovulation induction protocol; ultrasound scans were performed by the same qualified operator and HCG criteria was strictly followed. The same technical team also carried out laboratory evaluation and decision to undergo prolonged in vitro culture was done based on objective criteria. All this should minimize confounding factors.

Our perspective is to perform genetic studies using pre-implantation screening techniques to exclude aneuploidy embryos in this good response population. This may point us an $\mathrm{AMH}$ threshold that indicates a risk of aneuploidy.

\section{CONCLUSIONS}

Patients eligible to achieve blastocyst formation have higher levels of serum AMH; however, an excessively high concentration of this hormone can be harmful to blastocyst development.

\section{CONFLICT OF INTERESTS}

No conflict of interest have been declared.

\section{Corresponding author:}

Emily De Conto

UFRGS - Universidade Federal do Rio Grande do Sul

Porto Alegre/RS, Brazil

E-mail: emydconto@yahoo.com.br

\section{REFERENCES}

Anckaert E, Smitz J, Schiettecatte J, Klein BM, Arce JC. The value of anti-Mullerian hormone measurement in the long $\mathrm{GnRH}$ agonist protocol: association with ovarian response and gonadotrophin dose adjustments. Hum Reprod. 2012 Jun;27(6):1829-39.

Briggs R, Kovacs G, MacLachlan V, Motteram C, Baker HW. Can You Ever Collect Too Many Oocytes? Hum Reprod. 2015;30:81-7.

Broekmans FJ, Kwee J, Hendriks DJ, Mol BW, Lambalk CB. A systematic review of tests predicting ovarian reserve and
IVF outcome. Hum Reprod Update. 2006;12:685-718. de los Santos MJ, Mercader A, Galán A, Albert C, Romero $\mathrm{JL}$, Pellicer $\mathrm{A}$. Implantation rates after two, three, or five days of embryo culture. Placenta. 2003;24:S13-9.

Evans J, Hannan NJ, Edgell TA, et al. Fresh versus frozen embryo transfer: backing the clinical decisions with scientific and clinical evidence. Hum Reprod Update. 2014;20:808-21.

Fisch JD, Rodriguez H, Ross R, Overby G, Sher G. The Graduated Embryo Score (GES) predicts blastocyst formation and pregnancy rate from cleavage-stage embryos. Hum Reprod. 2001;16:1970-5.

Gardner DK, Surrey E, Minjarez D, Leitz A, Stevens J, Schoolcraft WB. Single blastocyst transfer: a prospective randomized trial. Fertil Steril. 2004;81:551-5.

Guerif F, Lemseffer M, Couet M, Gervereau O, Ract V, Royère $D$. Serum antimüllerian hormone is not predictive of oocyte quality in vitro fertilization. Ann Endocrinol (Paris). $2009 ; 70: 230-4$.

Kamel HM, Amin AH, Al-Adawy AR. Basal serum anti-Mullerian hormone $(\mathrm{AMH})$ is a promising test in prediction of occurrence of pregnancy rate in infertile women undergoing ICSI cycles. Clin Lab. 2014;60:1717-23.

Langley MT, Marek DM, Gardner DK, Doody KM, Doody KJ. Extended embryo culture in human assisted reproduction treatments. Hum Reprod. 2001;16:902-8.

Lie Fong S, Baart EB, Martini E, Schipper I, Visser JA, Themmen AP, de Jong FH, Fauser BJ, Laven JS. Anti-Müllerian hormone: a marker for oocyte quantity, oocyte quality and embryo quality? Reprod Biomed Online. 2008;16:66470 .

Lin WQ1, Yao LN, Zhang DX, Zhang W, Yang XJ, Yu R. The predictive value of anti-Mullerian hormone on embryo quality, blastocyst development, and pregnancy rate following in vitro fertilization-embryo transfer (IVF-ET). J Assist Reprod Genet. 2013;30649-55.

Satwik R, Kochhar M, Gupta SM, Majumdar A. Anti-mullerian hormone cut-off values for predicting poor ovarian response to exogenous ovarian stimulation in in-vitro fertilization. J Hum Reprod Sci. 2012;5:206-12. 
Sills ES, Collins GS, Brady AC, Walsh DJ, Marron KD, Peck AC, Walsh AP, Salem RD. Bivariate analysis of basal serum anti-Müllerian hormone measurements and human blastocyst development after IVF. Reprod Biol Endocrinol. 2011 2;9:153.

Smeenk JM, Sweep FC, Zielhuis GA, Kremer JA, Thomas $C M$, Braat DD. Antimüllerian hormone predicts ovarian responsiveness, but not embryo quality or pregnancy, after in vitro fertilization or intracyoplasmic sperm injection. Fertil Steril. 2007;87:223-6.

Steward RG, Lan L, Shah AA, Yeh JS, Price TM, Goldfarb JM, Muasher SJ. Oocyte number as a predictor for ovar- ian hyperstimulation syndrome and live birth: an analysis of 256,381 in vitro fertilization cycles. Fertil Steril. 2014;101:967-973.

Sunkara SK, Rittenberg V, Raine-Fenning N, Bhattacharya S, Zamora J, Coomarasamy A. Association between the number of eggs and live birth in IVF treatment: an analysis of 400.135 treatment cycles. Hum Reprod. 2011;26:176874.

van der Gaast $\mathrm{MH}$, Eijkemans MJ, van der Net JB, et al. Optimum number of oocytes for a successful first IVF treatment cycle. Reprod Biomed Online. 2006;13: 476-80. 